\title{
Compact-slab radio-frequency discharge CO laser
}

\begin{abstract}
Andrey Ionin, Andrey Kozlov, Leonid Seleznev, and
\end{abstract} Dmitry Sinitsyn

A novel repetitively pulsed first-overtone laser produces an average output power of $0.3 \mathrm{~W}$ and a laser efficiency of $0.5 \%$ at operating wavelengths of 2.5-4.0 $\mathrm{\mu m}$.

Carbon monoxide (CO) lasers ${ }^{1}$ are very attractive sources of mid-IR radiation for laser spectroscopy and multicomponentgas analysis. The spectral range $(2.5-4.2 \mu \mathrm{m})$ of first-overtone (FO) CO lasers (characterized by vibrational transitions from $V+2 \rightarrow V$ ) covers those of the well-established hydrogen fluoride and deuterium fluoride lasers, but with significantly smaller vibrational-rotational line spacings. Their full output spectra may contain more than 400 emission lines, ${ }^{2}$ many of which coincide with absorption lines exhibited by a wide range of organic and inorganic materials such as, $\mathrm{H}_{2} \mathrm{O}, \mathrm{CO}_{2}, \mathrm{CH}_{4}, \mathrm{NO}_{2}, \mathrm{NO}, \mathrm{SO}_{2}$, acetone, benzene, and methanol. FO CO lasers operating at these wavelengths can thus be used to assess the impact of resonances on various media in fields as diverse as, nonlinear spectroscopy, atmospheric remote sensing, and laser chemistry. FO CO lasers are among the best devices for laser-based spectroscopic analysis of multicomponent-gas mixtures. ${ }^{3}$ In addition, the longerwavelength section of their output spectrum coincides with the atmospheric 'transparency window.'

Successful applications require the development of compact, sealed-off FO CO lasers. Present-generation FO CO lasers use either fragile glass components, e.g., for continuous-wave (cw) gas-flow high-voltage DC discharge, or very complicated, largescale designs (e.g., for high-voltage electron-beam-sustained discharge or supersonic cooling), and are not suitable for integration in compact multiwavelength-laser gas analyzers. ${ }^{3}$ One of the most promising approaches to producing compact gas lasers uses radio-frequency (RF) discharge in a slab geometry to pump an active medium. We developed a cryogenically cooled slab RFdischarge CO laser ${ }^{4,5}$ (see Figure 1). Significant operational advantages of our FO CO laser include its slab RF-discharge pumping operation, robust compact stainless-steel design, sealedoff performance, cryogenic cooling of the electrode system
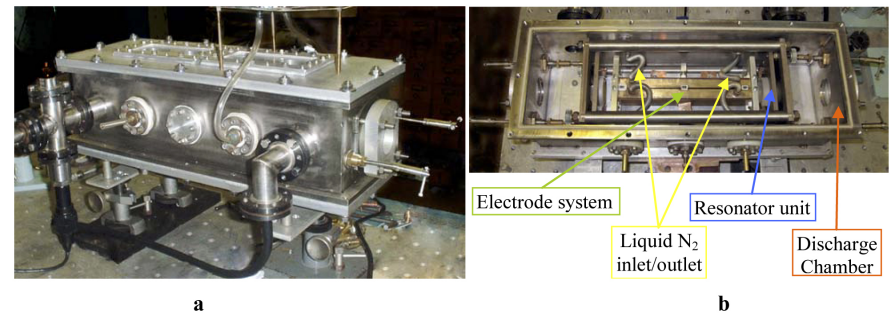

Figure 1. Slab radio-frequency discharge first-overtone carbon monoxide (CO)-laser setup. (a) General view. (b) Open view.

combined with discharge-chamber walls at room temperature, and a relatively low operational voltage.

The laser's active volume is $3 \times 30 \times 250 \mathrm{~mm}^{3}$ (the discharge chamber's total internal volume is $\sim 81$ ). The electrode system is cooled by liquid nitrogen to $\sim 120 \mathrm{~K}$ (using $\sim 5 \mathrm{~L} / \mathrm{hr}$ ). An RF power supply with a carrier frequency of 81 or $60 \mathrm{MHz}$, operating in a repetitively pulsed mode, is used for discharge ignition. The power supply produces up to $1000 \mathrm{~W}$ of peak-power rectangular pulses of variable duration, $\tau=(0.01-0.99) \times F^{-1}$, where $F=(100-500) \mathrm{Hz}$ is the pulse-repetition rate. The discharge chamber is filled with gas mixtures $\mathrm{CO}: \mathrm{O}_{2}: \mathrm{N}_{2}: \mathrm{He}$ of different component concentrations at a total gas pressure of 15-22Torr. Note that gas mixtures with high oxygen content are needed for stable long-term operation. ${ }^{4,5}$ We use a stable laser resonator with very-high-reflectivity mirrors (>99\% in the spectral range 2.5$4.2 \mu \mathrm{m})$. Two sets of mirrors optimized for either $2.5-4.2$ or 2.5$3.2 \mu \mathrm{m}$ operation can be used, depending on the requisite output spectrum, which strongly depends on the laser's output-mirror characteristics (see Figure 2).

The maximum FO CO-laser average output power obtained with the first laser-resonator mirror set was $\sim 0.32 \mathrm{~W}$ in the $\sim 2.55-3.15 \mu \mathrm{m}$ spectral range. The output spectrum included more than 40 vibrational-rotational lines, corresponding to overtone vibrational bands from $8 \rightarrow 6$ up to $23 \rightarrow 21$ (see the blue bars in Figure 2). The laser efficiency under these conditions

Continued on next page 


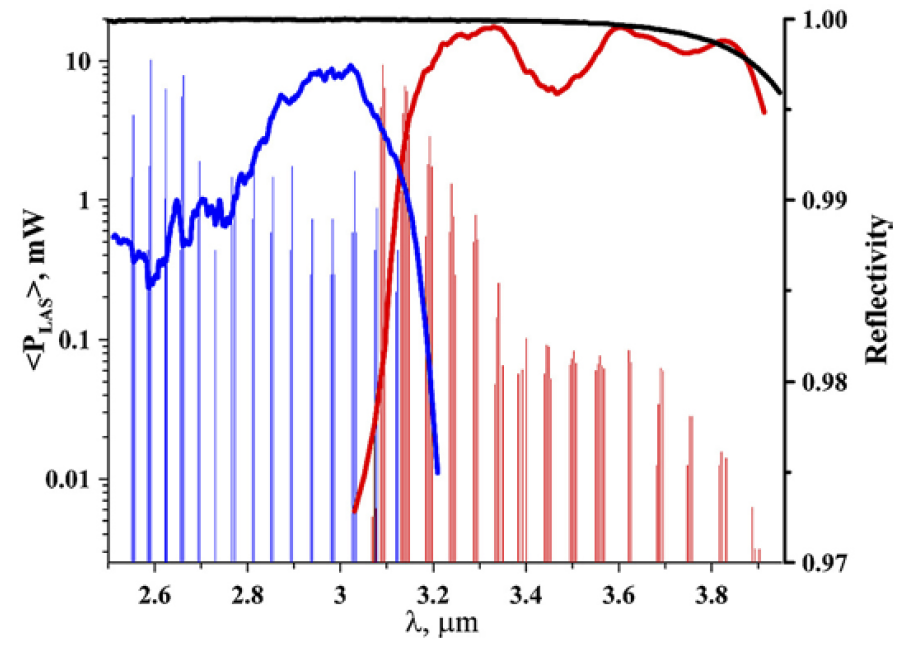

Figure 2. Vertical bars: Typical first-overtone CO-laser spectra obtained with two laser-resonator mirror sets (laser power, $P_{L A S}$, in $m W$ ). Curves: Characteristic reflectivity of the rear mirror (black) and the output mirrors for the first and second sets (blue and red, respectively).

was $\sim 0.5 \%$. Using the second set of mirrors, FO CO lasing was observed at $\sim 3.05-3.95 \mu \mathrm{m}$. The output spectrum consisted of more than 60 vibrational-rotational lines, corresponding to overtone vibrational bands from $22 \rightarrow 20$ up to $36 \rightarrow 34$ (see the red bars in Figure 2). The full spectrum obtained using both sets of resonator mirrors included more than 100 vibrational-rotational lines in the $2.5-4.0 \mu \mathrm{m}$ wavelength range, with a maximum single-line average output power of $\sim 12 \mathrm{~mW}$. Our next objective is to increase the laser efficiency to $\sim 5-10 \%$, which has been achieved in facilities using other types of electric discharges. ${ }^{2}$

\section{Author Information}

\section{Andrey Ionin, Andrey Kozlov, Leonid Seleznev, and}

\section{Dmitry Sinitsyn}

Gas Lasers Laboratory

P. N. Lebedev Physical Institute

Russian Academy of Sciences

Moscow, Russia

Andrey A. Ionin is chief scientist and head of the Gas Lasers Laboratory. His scientific interests focus on high-power gas lasers, including pulsed, repetitively pulsed, and $\mathrm{cw} \mathrm{CO}_{2}, \mathrm{CO}$, and $\mathrm{N}_{2} \mathrm{O}$ lasers, their applications, low-temperature plasma, and nonlinear optics. He has published more than 300 papers, eight patents, and two monographs, and worked in German and US laser centers.
Andrey Kozlov graduated from the Moscow Engineering and Physics Institute (Russia) in 2002. He is a junior scientist. His present research interests are in high-power gas lasers including pulsed, repetitively pulsed, and cw lasers, their applications, and low-temperature plasma. He has published more than 40 articles.

Leonid Seleznev is a senior scientist. His current research interests are in high-power gas lasers including pulsed, repetitively pulsed, and cw lasers, their applications, and low-temperature plasma. He has more than 100 publications including two patents.

Dmitry Sinitsyn is a senior scientist. His research interests focus on electrical-discharge molecular-gas lasers including pulsed, repetitively pulsed, and cw lasers, their applications, and lowtemperature plasma. He has more than 160 publications including three patents.

\section{References}

1. A. A. Ionin, Electric discharge CO lasers, M. Endo and R. Walter (eds.), Gas Lasers, p. 201, CRC Press, Boca Raton, FL, 2007.

2. N. Basov, G. Hager, A. Ionin, et al., Efficient pulsed first-overtone CO laser operating within the spectral range of 2.5-4.2 $\mu$ m, IEEE J. Quant. Electron. 36 (7), p. 810, 2000

3. O. G. Buzykin, A. A. Ionin, S. V. Ivanov, et al., Resonant absorption of first-overtone CO laser radiation by atmospheric water vapor and pollutants, Laser Part. Beams 18, p. $697,2000$.

4. A. A. Ionin, L. V. Seleznev, A. V. Shelestovich, and D. V. Sinitsyn, Repetitively pulsed and CW sealed-off slab CO laser with cryogenic cooling, Proc. SPIE 6454, p. 645406, 2007. doi:10.1117/12.698340

5. A. A. Ionin, A. Yu. Kozlov, L. V. Seleznev, and D. V. Sinitsyn, Slab overtone carbon monoxide laser, Proc. SPIE 7196, p. 71960A, 2009. doi:10.1117/12.808211 